\title{
Changes in sensorimotor functions after spinal lesions evaluated in terms of long-latency reflexes
}

\author{
H ACKERMANN, H C DIENER, J DICHGANS \\ From the Department of Neurology, University of Tubingen, FRG
}

SUMMARY Lesions of the central afferent and efferent pathways cause onset-delays of long-latency EMG responses from anterior tibial muscle after passive dorsiflection of the ankle joint in standing subjects. In 23 patients with spinal tumour or cervical stenosis and clinical signs of a medullary lesion, short-, medium- and long-latency EMG responses from distal leg muscles after ankle dorsiflection were recorded prior to and after surgical intervention. Fifteen of the patients were re-examined between 1 and 2 years after surgery. The results of the follow-up study support the hypothesis of a supraspinal pathway for long-latency EMG responses in distal leg muscles and show their significance as a quantitative measure of sensorimotor functions.

Up to now the objective evaluation of successful or failing neurosurgery in patients with spinal tumour, angioma or cervical myelopathy regarding motor and/or sensory functions had to rely on the well established and widely used recording of somatosensory evoked cerebral potentials (SEPs) after electrical stimulation of a peripheral nerve and the still experimental methods of percutaneous electrical or magnetic stimulation of the motor cortex with subsequent recording of EMG potentials. These procedures, however, suffer from several shortcomings; SEPs can only reliably identify lesions of the proprioceptive-afferent pathways. Stimulation of the tibial nerve at the ankle excites afferent fibres from the foot and ankle joint only, sparing input from muscle spindles situated above the ankle. Furthermore, SEPs are frequently abolished in patients with spinal lesions, although sensory functions are partially preserved, a fact that clearly limits a quantifiable followup. The analogous neurophysiological method to identify lesions of the central efferent pathways, the percutaneous stimulation of motor cortex with recording of evoked muscle action potentials, is still experimental, ${ }^{1-4}$ and there are several unresolved questions regarding this technique, as recently summarised by Young and Cracco. ${ }^{5}$

Address for reprint requests: Dr H Ackermann, Department of Neurology, University of Tubingen, Liebermeisterstr. 18-20 D-7400 Tubingen, FRG.

Received 16 July 1986 and in revised form 26 June 1987. Accepted 26 June 1987
EMG responses of longer latency which can be recorded in addition to the fast acting segmental stretch reflex after preloading, provide further information about the functional state of the spinal and supraspinal afferent and efferent pathways as a whole. ${ }^{6-15}$

This assumption is based on the following facts: the abrupt dorsiflection of the foot in upright standing subjects evokes EMG responses of short (SL) and medium latency (ML) in the stretched triceps surae and a long-latency (LL) response in the antagonistic anterior tibial muscle. This experimental paradigm in comparison with similar studies on the arm has the advantage that ML and LL appear in antagonistic muscles and therefore are clearly separable. ${ }^{1617} \mathrm{SL}$ corresponds to the segmental stretch reflex. Previous studies have indicated that under our experimental conditions $M L$ and $L L$ are mediated by different pathways. ML represents a polysynaptic spinal reflex, most probably mediated by group II afferents to the spinal cord, whereas the "reflex pathway" of LL includes supraspinal structures. Patients with spinal or cerebral lesions show a significant delay of $\mathrm{LL}$. ML is missing in up to $50 \%$ of these recordings. The latency of ML, if present, is unaltered compared with normals. ${ }^{1415}$

Physiology and anatomical pathways of longlatency EMG responses are speculative up to now. If the assumption of a transcortical loop is valid, the recording of $\mathrm{LL}$ responses offers a possibility to investigate and monitor both the afferent and efferent part of the "reflex" pathway. Studies in patients with 
lesion of known location should ideally lead to a better understanding of the physiology and anatomy of long-loop reflexes and result in objective parameters of sensorimotor functions in the follow-up of the disease.

In order to assess the reliability of EMG responses of long latency as an atraumatic way to monitor objectively both sensory and motor functions, we recorded these EMG potentials in a group of patients with spinal lesions prior to and once or twice after surgical intervention. The latencies of $L L$ were related to a clinical score measuring sensorimotor functions.

As far as we know there is no systematic follow-up study of the time-course of long latency reflexes in patients who underwent surgical treatment. Only the time-course of somatosensory evoked potentials after electrical stimulation of the posterior tibial nerve has been studied in patients with cervical stenosis before and after surgical decompression of the spinal cord. ${ }^{18}$

\section{Methods}

\section{EMG responses}

Patients stood upright with their feet parallel on a force measuring platform which was suddenly rotated around the axis of the ankle joint. Subjects were not allowed to use their arms for support. Platform tilts toe-up with a velocity of 50 degrees/second and an amplitude of $4^{\circ}$ were used as mechanical stimulus. By means of surface electrodes, EMGs were recorded from the triceps surae and anterior tibial muscles, on both sides consecutively. EMG signals were rectified, filtered, and amplified (bandpass $1.6 \mathrm{~Hz}-1 \mathrm{kHz}$ ). Eight platform tilts were applied to each patient. The time interval between single platform tilts varied randomly between 5 and 12 seconds. The onset of the EMG activity was visually identified on a computer display. EMG latencies were measured in terms of the time elapsed between the signal triggering tilt of the platform and the onset of EMG activity. This was done separately for each of the eight corresponding runs by visual identification of the onset of EMG activity on the graphics terminal of the computer. In order to avoid a bias all latency measurements were done independently by a technician not familiar with the expected results. Integrals of SL and ML were calculated from the averaged EMG activity. The integral of LL was not taken into account, because it was impossible in about $50 \%$ of the recordings to clearly determine the end of $L L$.

\section{Patients}

Twenty three patients were investigated in whom a spinal lesion was demonstrated by neuroradiological methods (myelography, computed tomography, spinal angiography, and/or magnetic resonance imaging). All patients underwent surgical treatment. Thirteen patients had a cervical stenosis with chronic myelopathy (nuclear disc prolapse and/or spondylosis), three patients suffered from syringomyelia, three from spinal angioma and four from an extramedullary spinal tumour (neurinoma or meningioma). EMG responses were recorded in all but one patient within a 4 week period before surgical intervention. A second recording was made in all but one patient within 2 to 14 weeks after surgery. Fifteen patients were re-examined a third time between 1 or 2 years after surgical treatment. SEPs were recorded in 21 patients prior to surgery, in 14 patients at the time of the first and in 13 patients at the second electromyographic investigation.

\section{Clinical score}

Only patients with sensory (proprioception) and/or motor symptoms in one or both legs were included in the study. The degree of impairment of sensorimotor functions was assessed by means of a clinical score, explained in table 1 , separately for both legs and separately for sensory and motor symptoms.

\section{Normal controls}

Normal data were collected from a group of $\mathbf{4 0}$ persons without neurological disease (median of age 38 years, arithmetic mean 41.3 years, 20 men and 20 women). In order to investigate test-retest reliability of our method, we recorded SL, ML, and LL responses from both legs in a population of 20 normals (aged 20-35 years) twice within a period of 2 weeks.

\section{Statistics}

For statistical analysis we used the $t$ test for related samples and a one way analysis of variance for related samples using a mathematical correction for incomplete numbers. The level of significance was set at $5 \%$.

\section{Results}

Time course of EMG latencies

The reliability study in our group of normal subjects revealed no statistically significant differences either in latency of the three EMG responses or in integral of SL and ML within a 2 week period. The difference in mean latencies from the first to the second examination was $1.7 \mathrm{~ms}$ for SL, $3.6 \mathrm{~ms}$ for $\mathrm{ML}$ and $6.7 \mathrm{~ms}$ for $L L$.

Eighteen of the $44 \mathrm{LL}$ recordings $(=41 \%)$ prior to

\section{Table 1 Clinical score}

\section{Score of sensory impairment}

$0=$ normal vibration sense (7-8/8) at the big toe and ankle joint, normal joint position sense at the basic joint of the big toe and ankle joint

$1=$ mild decrease in vibration sense $(>3 / 8)$

$2=$ marked decrease $(<3 / 8)$ or loss of vibration sense

3 = mild impairment of joint position sense with/without decrease of vibration sense

4 = marked impairment of joint position sense (no detection of larger joint movements) with/without decrease of vibration sense

\section{Score of motor impairment}

$0=$ no impairment of motor functions in toe, foot and leg muscles

1 = exaggerated tendon reflexes with/without clonus

2 = positive extensor response and/or spasticity

3 = mild paresis of leg muscles (active movements against gravity possible)

4 = moderate to marked paresis of leg muscles 
surgery showed a delay of $L L$, in two cases $(=5 \%)$ LL was missing. In 24 recordings $(=54 \%)$ the latency was within the normal range (arithmetic mean (AM) +2.5 standard deviations (SD)). Mean latencies of SL, ML and LL from the patients prior to and after surgical treatment are shown in table 2 . There was no significant difference in latency and integral of SL along time. ML was absent in $48 \%$ of the recordings prior to surgery $(17 \%$ in normals), and sometimes reappeared postoperatively. At the second investigation after surgery ML was missing in $30 \%$ of the recordings. This partially explains the significant increase of ML integrals 1 to 2 years after surgery. The mean latency of ML, if the response was initially seen, did not change after surgery. This contrasts with a significant decrease of the latency $L L$ after surgical decompression of the spinal cord (fig 1). Eleven out of the 17 recordings with originally delayed latency were within normal range at the first postsurgical investigation, and all but one recording resulted in a normal latency 1 to 2 years after operation (fig 2). LL remained abolished in the two legs with missing $\mathrm{LL}$ before surgery.

Prior to surgery we found in 10 patients a marked side-difference of sensory and/or motor functions between the two legs with only slight impairment at the less affected leg. The latency of $L L$ was significantly longer on the more affected side and the shortening of the latency after surgery also more pronounced compared with the changes at the less affected leg (table 3 ).

\section{Latencies of $L L$ and clinical signs}

Prior to surgery the mean score of motor signs for the recordings with pathologically delayed LL (AM + $2 \cdot 5 \mathrm{SD},=170 \mathrm{~ms}$ ) amounted to $2 \cdot 1$, the mean score of sensory signs to $2 \cdot 7$. Five recordings were from legs with pure sensory signs, a fact which explains the relatively small motor score in this group. For the recordings with latencies of $L L$ less than $A M+1 S D$ $(=147 \mathrm{~ms})$ the mean score of motor signs at the corre- sponding legs was $1 \cdot 5$, and the mean score of sensory signs $\mathbf{1} \cdot \mathbf{2}$. For the remaining recordings with still normal latency, but greater than AM + $1 \mathrm{SD}$, the mean score of motor signs at the corresponding legs amounted to $2 \cdot 1$, of sensory signs to $1 \cdot 3$.

At the time of the first postsurgical recording no patient had deteriorated sensory and/or motor symptoms compared with the presurgical examination. Twenty of the 23 patients were clearly improved. Three out of six recordings (one from each leg) in the remaining three patients showed a moderate increase of latency, but still within the limits of our normal population (less than $1 \mathrm{SD},=15 \mathrm{~ms}$ ). In 19 out of the 20 patients with improvement of sensory and motor symptoms after surgical decompression of the spinal cord a concomitant decrease of latency could be observed. The remaining patient showed a small increase of latency despite a slight clinical improvement. In two patients, one suffering from marked spastic paresis and moderate disturbances of joint position sense, the other with severe impairment of dorsal columns function, the LL response was abolished in the clinically more affected leg. Despite some clinical improvement, LL was still missing after surgical intervention (fig 2 ).

Seven recordings documented an initial decrease of LL at the first postsurgical examination, but a later increase of latency at the second investigation 1 to 2 years after surgery. Since the latencies of $L L$ were not pathologically delayed before surgery, these recordings are not included in fig 2. Two of these seven recordings were obtained from a patient who suffered a second cervical disc prolapse with worsening of clinical signs. In another patient a relapse of an originally successfully operated spinal angioma was diagnosed clinically. The latency of the long-latency EMG potential increased again at one side, on the other $\mathrm{LL}$ was missing throughout follow-up (fig 2). In a third patient the increase of latency at both legs could be attributed to a recently superimposed polyneuropathy, due to treatment with cytostatics (fig 2). In one patient clinical deterioration took place 3 years

Table 2 Mean values and standard deviations (SD) of EMG latencies and integrals from short (SL), medium (ML), and long latency $(L L)$ responses

\begin{tabular}{|c|c|c|c|c|c|c|c|}
\hline \multirow[b]{2}{*}{ Subjects } & \multirow{2}{*}{$\begin{array}{l}\text { Date of } \\
\text { recording }\end{array}$} & \multirow{2}{*}{$\begin{array}{l}\text { Number } \\
\text { of } \\
\text { recordings }\end{array}$} & \multicolumn{3}{|c|}{ Latency (ms) } & \multicolumn{2}{|c|}{ Integral $(\mu V s)$} \\
\hline & & & $S L$ & $M L$ & $L L$ & $S L$ & $M L$ \\
\hline Normals & & 80 & $48.9(6.0)$ & $89 \cdot 5(10 \cdot 0)$ & $132.0(15.0)$ & $1.81(0.8)$ & $3.43(2.8)$ \\
\hline \multirow[t]{3}{*}{ Patients } & \multirow{3}{*}{$\begin{array}{l}\text { Before surgery } \\
2-14 \text { weeks after } \\
\text { surgery } \\
1-2 \text { years after } \\
\text { surgery }\end{array}$} & 44 & $43 \cdot 9(6 \cdot 3)$ & $76.4(9.4)$ & $172 \cdot 2(35 \cdot 5)^{*}$ & $4.05(2.3)^{*}$ & $1 \cdot 06(1 \cdot 3)$ \\
\hline & & 44 & $44 \cdot 6(8 \cdot 7)$ & $76.8(9 \cdot 8)$ & $152.0(29.6)^{*}$ & $4 \cdot 36(3 \cdot 1)$ & $1.64(1.9)$ \\
\hline & & 30 & $43.9(6.8)$ & $73 \cdot 5(7 \cdot 9)$ & $148.9(16.6)^{*}$ & $4 \cdot 67(2 \cdot 1)$ & $2.12(1.9)^{*}$ \\
\hline
\end{tabular}

* Significant difference, $\mathrm{p}<0.05$. 


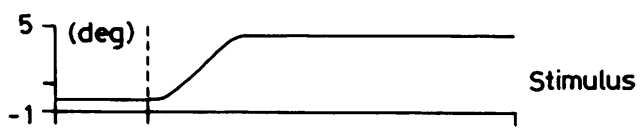

(a) Before surgery
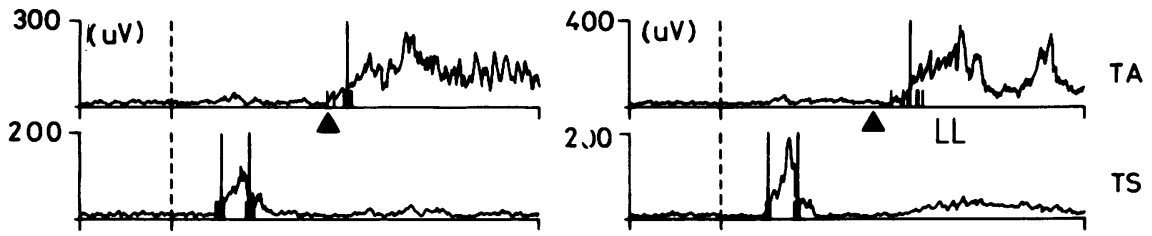

(b) After surgery
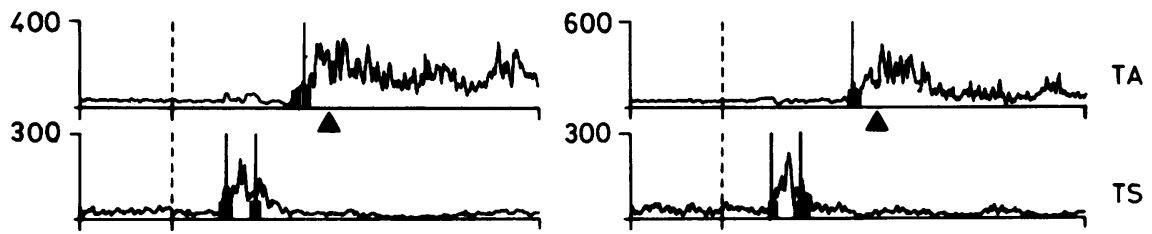

(c) Three years later
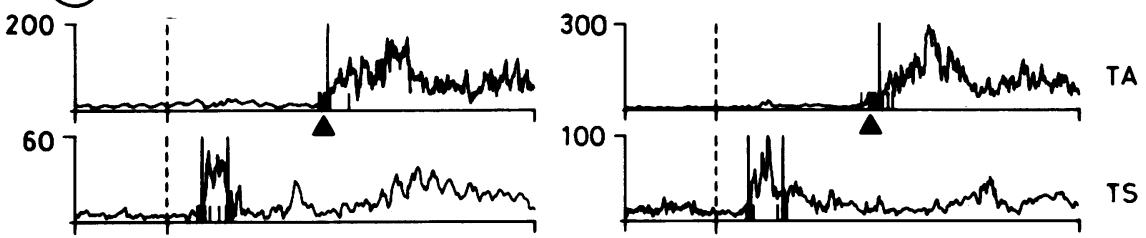

(d) After re-operation

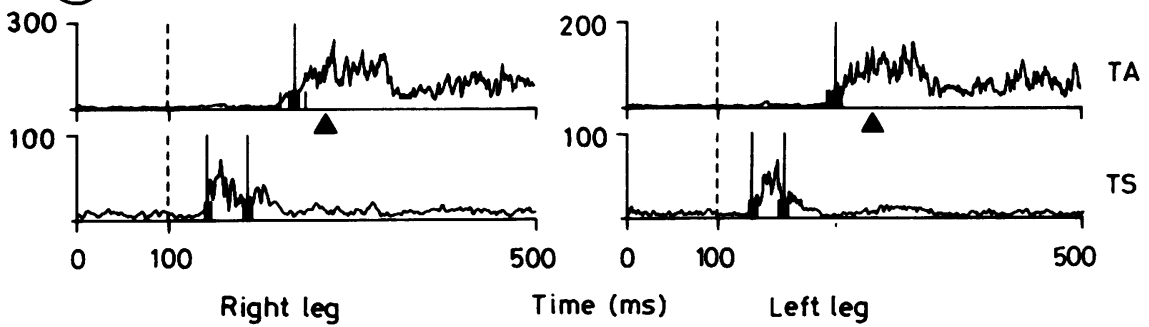

Fig 1 Follow-up of a patient with paraparesis and impairment of vibration and joint position sense at both legs. Angiography revealed a spinal angioma (Th 10). Latencies of EMG responses of long latency $(L L)$ in anterior tibial muscle (TA): $191 \mathrm{~ms}$ in the right leg, $208 \mathrm{~ms}$ in the left leg $(A M+2 \cdot 5 S D$ in normals: $170 \mathrm{~ms}$, arrow $)$. After surgical intervention a marked clinical improvement could be noted with concomitant decrease of $L L$. Latency 2 years after surgery: $144 \mathrm{~ms}$ in the right leg, $141 \mathrm{~ms}$ in the left $(B)$. Three years after surgery clinical deterioration with parallel increase in latencies to $177 \mathrm{~ms}$ (right leg) and $182 \mathrm{~ms}$ (left leg) respectively (C). Surgical exploration of the spinal cord revealed compression of the medulla by scar tissue. After extirpation, clinical signs improved rapidly. Normal latencies: $131 \mathrm{~ms}$ in the left leg, $139 \mathrm{~ms}$ in the right leg (D). The short-latency response (SL) remained unchanged throughout follow-up. The medium-latency response (ML) in triceps surae (TS) was very small and could only be detected in single trials. The vertical dashed line indicates the beginning of platform-tilt. Mean latencies of eight trials are marked by vertical solid lines, short lines indicate latencies of single trials. 


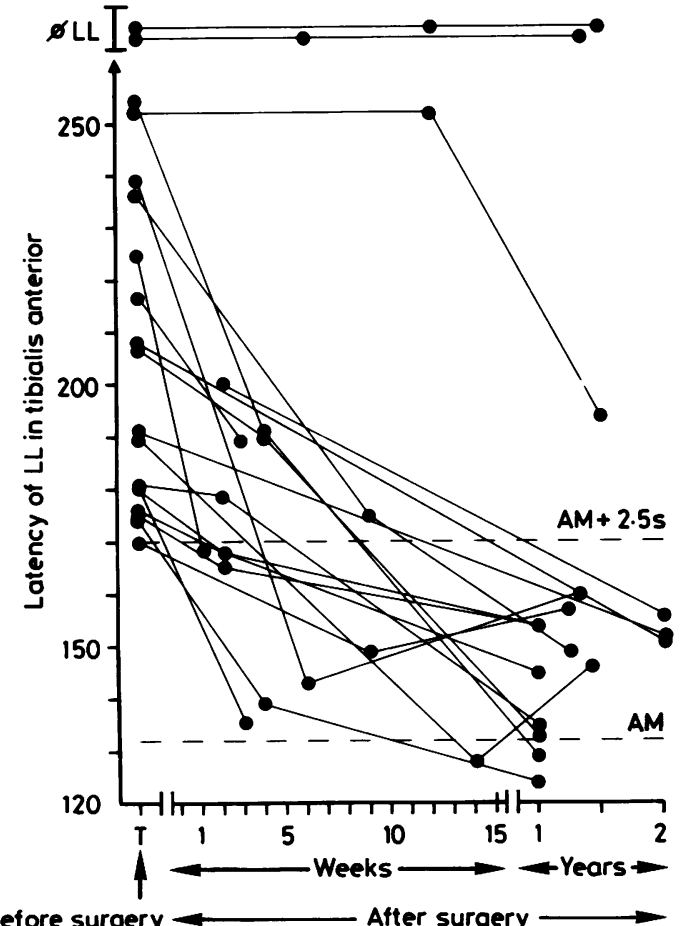

Fig 2 Follow-up of EMG responses of long latency ( $L L)$ after surgery: included are recordings $(n=20$, out of 46 recordings) with pathologically delayed latency $(n=17)$ or missing $L L(n=2)$ and one recording of a patient with two postsurgical measurements only, but pathological latency at the first postsurgical investigation. $A M=$ arithmetic mean of latencies in the group of normal subjects $(n=50)$, upper limit of normality $(A M+2 \cdot 5 S D)=170 \mathrm{~ms}$.

after surgery (see fig 1). But 2 years after surgery LL was still normal, therefore the increase of latency is not shown in fig 2 .

Clinical scores and long-latency EMG responses of those patients in whom long-term recordings after 1 to 2 years post-surgery were available ( 15 patients, 30 recordings) are compared in fig 3 . Included are only recordings $(n=23)$ from those legs in which a change of clinical signs of at least on score could be detected. Figure 3 indicates the parallel course of severity of clinical signs and LL (latencies at score 4 are significantly higher than at score 1 or 0 ), but also the close relationship between clinical improvement and decrease in latency. In one patient only an increase of the latency of the long-latency EMG potential could be detected (15 ms compared with pre-surgery) despite unchanged clinical score. The remaining six recordings were unchanged in respect to both clinical score and LL.

\section{Discussion}

Sudden stretch of a muscle under certain circumstances, for example, with preloading of the muscle and instruction to the subject to resist the imposed perturbation, evokes besides a fast acting and presumably monosynaptic spinal stretch reflex, one or more EMG responses of longer latency. Origin and pathway of the latter are still a matter of controversy. There is some evidence, both from clinical and experimental investigations, that in humans transcortical loop mechanisms contribute to load compensation following perturbation of forearm muscles (for review see refs 13, 19). Concerning EMG responses of longer latency recorded from distal leg muscles following passive ankle dorsiflection ${ }^{1120}$ or stance and gait perturbations in a treadmill paradigm, ${ }^{12-23}$ the assumption of a transcortical loop is, however, questioned and multiple successive Ia- and II-bursts or a polysynaptic spinal transmission have been suggested.

The present study examined the timing of reflex responses in the anterior tibial and triceps surae muscles after dorsiflection of the ankle during standing in patients with spinal lesion (tumour or cervical myelopathy). The results demonstrate the increase in latency of long-latency EMG potentials in patients with spastic paresis and/or impairment of proprioception and the decrease in latency parallel to the improvement of clinical symptoms after surgery.

Admittedly, these findings are no proof of a transcerebral reflex pathway. ${ }^{24}$ The time-interval neces-

Table 3 Means and standard deviations (SD) of EMG responses of short (SL), medium (ML), and long latency (LL) from the clinically more and less affected leg

\begin{tabular}{|c|c|c|c|c|c|c|c|}
\hline \multirow{2}{*}{$\begin{array}{l}\text { Date of } \\
\text { recording }\end{array}$} & \multirow{2}{*}{$\begin{array}{l}\text { Number of } \\
\text { recordings }\end{array}$} & \multicolumn{3}{|c|}{ Latency (ms) more affected leg } & \multicolumn{3}{|c|}{ Less affected leg } \\
\hline & & $S L$ & $M L$ & $L L$ & $S L$ & $M L$ & $L L$ \\
\hline \multirow{3}{*}{$\begin{array}{l}\text { Before surgery } \\
2-14 \text { weeks after } \\
\text { surgery } \\
1-2 \text { years after } \\
\text { surgery }\end{array}$} & 20 & $46 \cdot 9(10 \cdot 3)$ & $83 \cdot 0(12 \cdot 1)$ & $190.0(28.0)^{*}$ & $46 \cdot 7(7 \cdot 3)$ & $85 \cdot 8(6 \cdot 2)$ & $157.9(14.5)$ \\
\hline & 20 & $48 \cdot 8(9 \cdot 6)$ & $82 \cdot 7(5 \cdot 5)$ & $157 \cdot 3(22 \cdot 9)$ & $45 \cdot 0(8 \cdot 6)$ & $83 \cdot 5(4 \cdot 9)$ & $139.4(16.9)$ \\
\hline & 10 & $49 \cdot 4(6 \cdot 4)$ & $79 \cdot 0(10 \cdot 1)$ & $146.8(13.8)^{*}$ & $44 \cdot 8(6 \cdot 7)$ & $75 \cdot 0(12 \cdot 0)$ & $147 \cdot 8(11 \cdot 8)$ \\
\hline
\end{tabular}

*Significant difference, $\mathrm{p}<0.05$. 


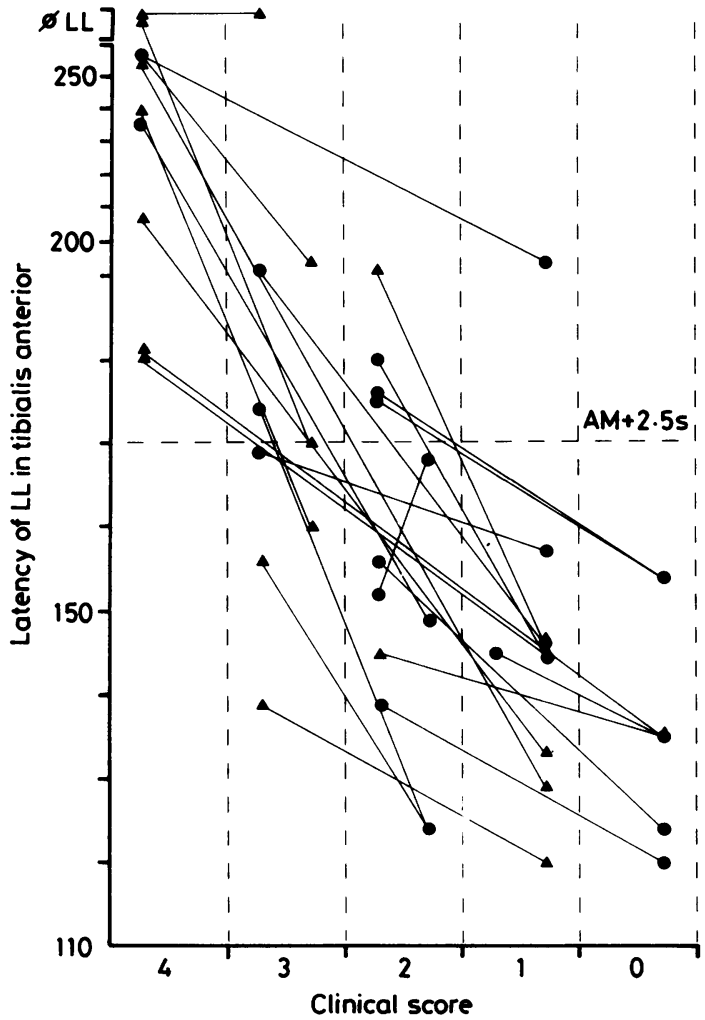

Fig 3 Comparison of latencies of long-latency EMG responses and clinical scores of those patients $(n=12$, out of 23 patients) with long-term follow-up (measurement 1 up to 2 years after surgery), separately for both legs and separately for sensory (triangles) and motor (dots) functions. Included are the legs with changes of sensory and/or motor functions of at least one score. The differences of latencies between presurgical and postsurgical measurement in the legs without clinical change were in all but one case less than $15 \mathrm{~ms} \quad(=1$ $S D)$.

sary for polysynaptic spinal transmission depends upon facilitatory influences of supraspinal structures, and loss of facilitation results in a delay of polysynaptic spinal reflexes. ${ }^{25}$ Both the flexor reflex recorded from anterior tibial muscle following electrical stimulation of medial plantar nerves in subjects with hemiparesis ${ }^{26} 27$ and the compensatory muscle activation of tibialis anterior following displacement in patients with spastic hemi- or paraparesis show a delay in latency. ${ }^{28}$ The increase in latency of the flexor reflex amounted up to $250 \mathrm{~ms}$. In the balancing paradigm the latency increased by 20 to $30 \mathrm{~ms} .{ }^{28}$ The electrically evoked flexor reflex from anterior tibial muscle and the mechanically evoked compensatory activation of distal leg muscles on a treadmill, both have a latency of 60 to $70 \mathrm{~ms}$. In contrast, the long- latency EMG response in our paradigm showed a mean latency in normals of $132 \mathrm{~ms}$. Therefore it is hardly conceivable that LL could be mediated in the same way as the polysynaptic spinal reflexes.

There is some indirect evidence supporting the assumption of a transcerebral loop in long-latency EMG responses after platform tilts toe-up. In upright standing normal subjects platform tilts toe-up evoke a cerebral potential $(\mathrm{Cz}-\mathrm{Fz})$ with a mean onset-latency of about $52 \mathrm{~ms}$ and a mean peak-latency of about $76 \mathrm{ms.}^{2930}$ These mechanically evoked cerebral potentials indicate that there is under our experimental conditions a powerful afferent input to cerebral structures with time characteristics compatible with a transcerebral loop. Furthermore, in children the EMG responses of short, medium and long latency following platform tilts toe-up show a different course during maturation. ${ }^{31}$

The differences of LL compared with muscular responses which represent polysynaptic spinal reflexes raise the question, whether $L L$ is a voluntary action. Several features of LL indicate that this response must be considered as "reflex like activity". In normal subjects the latency of LL is not influenced by attention and motor learning as it is the case for voluntaryo actions. The small standard deviation of the latencieso of $L L$ responses between consecutive trials in norma subjects $(=15 \mathrm{~ms})$ compared with a standard devi $\Omega$ ation of more than $42 \mathrm{~ms}$ in a reaction time paradigm with voluntary extension of the foot after comparable sensory stimuli is another argument against the assumption of voluntary action. ${ }^{17}$ In the present study the standard deviation of the latencies of the long-latency EMG responses was higher than $20 \mathrm{~ms}$ in five recordings. These latter cases which also revealed the most remarkable delay of LL may reflect the muscular responses of patients in whom LL represents a voluntary action because of a loss of the reflex response.

The delay of $L L$ in patients with a spinal lesion and impairment of sensorimotor functions at the legs argues against the assumption that $L L$ is a simple "shortening reaction" $32-34$ of the anterior tibial muscle after passive dorsiflection of the foot. In patients with spastic paresis the shortening reaction is missing or decreased in integral, but the latency of the shortening reaction remains unchanged. ${ }^{34}$

It is conceivable that the EMG potential of medium latency after platform tilts toe-up represents a polysynaptic spinal reflex. ML has a latency comparable to that of the compensatory activation of distal leg muscles and to the flexor reflex recorded from anterior tibial muscle. ${ }^{2728}$ Considering the recordings with sustained ML, the latency remained unchanged before and after surgical decompression of the spinal cord, supporting the assumption of different gener- 
ation of ML and LL. ${ }^{14} 15$

Clinical and experimental data suggest that there are some distinct neurophysiological mechanisms for positive symptoms, namely increase of phasic stretch reflexes and muscle tone, and negative symptoms (that is, paresis and impaired motor performance), within the syndrome of spasticity. The negative symptoms of the upper motor neuron syndrome are supposed to be the result of diminished input to pertinent alpha-motor neurons. ${ }^{3536}$ In agreement with this assumption, we observed that patients with a spastic paresis have a delay of $\mathrm{LL}$, whereas patients with marked spastic hypertonia but intact muscle force had normal latencies.

Notwithstanding the uncertainties about origin and pathway of long-latency EMG responses, the recording of LL may provide useful diagnostic information. The "hit rate" in detecting lesions prior to surgery amounted to about $46 \%$ of pathological findings for recordings of $\mathrm{LL}$, and the latency of $\mathrm{LL}$ had a high correlation with the clinical data. Thus, the present study demonstrates that the time-course of LL allows documentation of follow-up of sensorimotor signs after surgical intervention at the spinal cord.

Furthermore, the present paper indicates some superiority of measurements of long-latency EMG responses compared with somatosensory evoked cerebral potentials after electrical stimulation of the posterior tibial nerve (SEP) for the evaluation of clinical changes prior to and after surgery. The belief in the superiority of $\mathrm{LL}$ recordings is based upon three observations: (1) In 17 out of 42 SEP recordings before surgery the primary cortical potential (P40) was missing and therefore did not allow for quantitative measurement of sensory impairment. Only in eight of the 17 recordings did P40 recover after surgery. (2) In five out of 19 patients with follow-up of SEP recordings (at least two measurements) the P40 latency remained unchanged despite clinical improvement or increased without concomitant clinical deterioration. (3) In three out of four patients with relapse of the spinal lesion P40-latency did not reflect the clinical deterioration noted at the second investigation post-surgery. The clinical deterioration caused by relapse of the spinal lesion was reflected in all patients by $\mathrm{LL}$ measurements.

This is not surprising if we assume that LL is mediated by a "reflex loop" which includes central afferent as well as descending motor pathways and therefore should be affected in cases of both proprioceptive and motor disturbances. Additionally, in this study longlatency EMG potentials were evoked by means of stance perturbations, and this "natural" stimulation may provide more adequate information about the functional capacity of the sensorimotor system than artificial electrical stimulation of a peripheral nerve trunk.

This work was supported by the Deutsche Forschungsgemeinschaft SFB 307/A3. We thank JMJ Allum and V Dietz for helpful comments, M Bacher, H Rapp, and B Guschlbauer for technical support.

\section{References}

1 Merton PA, Morton HB. Electrical stimulation of the human motor and visual cortex through the scalp. J Physiol (Lond) 1980;305:9-10P.

2 Marsden CD, Merton PA, Mrrton HB. Percutaneous stimulation of the spinal cord and brain: pyramidal tract conduction velocities in man. J Physiol (Lond) 1982;328:6P.

3 Marsden CD, Merton PA, Morton HB. Direct electrical stimulation of corticospinal pathways through the intact scalp in human subjects. In: Desmedt JE, ed. Motor Control Mechanisms in Health and Disease. Advances in Neurology, vol. 39. New York: Raven Press, 1983:387-91.

4 Cowan JMA, Dick JPR, Day BL, Rothwell JC, Thompson PD, Marsden CD. Abnormalities in central motor pathway. Lancet 1984;ii:304-7.

5 Young RR, Cracco RQ. Clinical neurophysiology of conduction in central motor pathways. Ann Neurol 1985;18:606-10.

6 Phillips CG. Motor apparatus of the baboon's hand. The Ferrier Lecture. Proc R Soc Biol 1969;173:141-74.

7 Lee RG, Tatton WG. Motor responses to sudden limb displacements in primates with specific CNS lesions and in human patients with motor system disorders. Can J Neurol Sci 1975;2:285-93.

8 Wiesendanger M, Ruegg DG, Lucier GE. Why transcortical reflexes? Can J Neurol Sci 1975;2:295-301.

9 Marsden CD, Merton PA, Morton HB. Stretch reflex action in a variety of human muscles. J Physiol (Lond) 1976;259:531-60.

10 Gottlieb GL, Agarwal GC. Response to sudden torques about the ankle in man. II. Postmyotatic reactions. J Neurophysiol 1980;43:86-101.

11 Berardelli A, Hallett M, Kaufman C, Fine E, Berenberg W, Simon SR. Stretch reflexes of triceps surae in normal man. J Neurol Neurosurg Psychiatry 1982;45:513-25.

12 Chan CWY, Kearney RE. Is the functional stretch response servo controlled or preprogrammed? Electroencephalogr Clin Neurophysiol 1982;53:310-24.

13 Marsden CD, Rothwell JC, Day BL. Long-latency automatic responses to muscle stretch in man: Origin and function. In: Desmedt JE, ed. Motor Control Mechanisms in Health and Disease. Advances in Neurology, vol. 39. New York: Raven Press, 1983:509-39.

14 Diener HC, Dichgans J, Huelser PJ, Buettner UW, Bacher M, Guschlbauer B. The significance of delayed long-loop responses to ankle displacement for the diagnosis of multiple sclerosis. Electroencephalogr Clin Neurophysiol 1984;57: 336-42.

15 Diener HC, Ackermann H, Dichgans J, Guschlbauer B. Mediumand long-latency responses to displacements of the ankle joint in patients with spinal and central lesions. Electroencephalogr Clin Neurophysiol 1985;60:407-16.

16 Diener HC, Bootz F, Dichgans J, Bruzek W. Variability of postural "reflexes" in humans. Exp Brain Res 1983;52:423-8.

17 Diener HC, Dichgans J, Bootz F, Bacher M. Early stabilization of human posture after a sudden disturbance: influence of rate and amplitude of displacement. Exp Brain Res 1984;56:126-34.

$18 \mathrm{Yu} \mathrm{YL}$, Jones SJ. Somatosensory evoked potentials in cervical 
spondylosis. Correlation of median, ulnar and posterior tibial nerve responses with clinical and radiological findings. Brain 1985;108:273-300.

19 Chan CWY. Segmental versus suprasegmental contributions to the long-latency stretch responses in man. In: Desmedt JE, ed. Motor Control Mechanisms in Health and Disease. Advances in Neurology, vol. 39. New York: Raven Press, 1983:467-87.

20 Berardelli A, Sabra AF, Hallett M, Berenberg W, Simon SR. Stretch reflexes of triceps surae in patients with upper motor neuron syndromes. J Neurol Neurosurg Psychiatry 1983;46: $54-60$.

21 Berger W, Dietz V, Quintern J. Corrective reactions to stumbling in man: neuronal co-ordination of bilateral leg muscle activity during gait. J Physiol (Lond) 1984;357:109-25.

22 Dietz V, Quintern J, Berger W. Corrective reactions to stumbling in man: functional significance of spinal and transcortical reflexes. Neurosci Lett 1984;44:131-5.

23 Dietz V, Quintern J, Berger W. Afferent control of human stance and gait: evidence for blocking of group I afferents during gait. Exp Brain Res 1985;61:153-63.

24 Marsden CD, Merton PA, Morton HB, Adam J. The effect of lesions of the central nervous system on long-latency stretch reflexes in the human thumb. In: Desmedt JE, ed. Cerebral motor control in man: long loop mechanisms. Prog Clin Neurophysiol, vol. 4. Basel: Karger, 1978:334-41.

25 Baldissera F, Hultborn H, Illert $\mathrm{M}$. Integration in spinal neuronal systems. In: Brookhart JM, Mountcastle VB, eds. Handbook of Physiology. The nervous system II. Motor Control, Part I. Bethesda: American Physiological Society, 1981:509-95.

26 Meinck HM, Benecke R, Kuester S, Conrad B. Cutaneomuscular (flexor) reflex organization in normal man and in patients with motor disorders. In: Desmedt JE, ed. Motor Control Mechanisms in Health and Disease. Advances in Neurology, vol. 39.
New York: Raven Press, 1983:787-96.

27 Meinck HM, Kuester S, Benecke R, Conrad B. The flexor reflex-influence of stimulus parameters on the reflex response. Electroencephalogr Clin Neurophysiol 1985;61:287-98.

28 Dietz V, Berger W. Interlimb coordination of posture in patients with spastic paresis. Impaired function of spinal reflexes. Brain 1984;107:965-78.

29 Ackermann H, Diener HC, Dichgans J. The diagnostic significance of long-latency-reflexes and mechanically evoked cerebral potentials in spinal and cerebral lesions. $J$ Neurol (suppl) 1985;232:254.

30 Ackermann H, Diener HC, Dichgans J. Mechanically evoked cerebral potentials and long-latency muscle responses in the evaluation of afferent and efferent long-loop pathways in humans. Neurosci Lett 1986;66:233-8.

31 Haas G, Diener HC, Bacher M, Dichgans J. Development of postural control in children. Short-, medium-, and long-latency EMG responses of leg muscles after perturbation of stance. Exp Brain Res 1986;640:127-32.

32 Katz R, Rondot P. Muscle reactions to passive shortening in normal man. Electroencephalogr Clin Neurophysiol 1978;45:90-9.

33 Angel RW. Muscular contractions elicited by passive shortening. In: Desmedt JE, ed. Motor Control Mechanisms in Health and Disease. Advances in Neurology, vol. 39. New York: Raven Press, 1983:555-63.

34 Berardelli A, Hallett M. Shortening reaction of the tibialis anterior muscle. Electroencephalogr Clin Neurophysiol 1984;57:80P.

35 Walshe FMR. Contributions of John Hughlings Jackson to neurology: A brief introduction to his teachings. Arch Neurol 1961:5:119-31.

36 Sahrmann SA, Norton BJ. The relationship of voluntary movement to spasticity in the upper motor neuron syndrome. Ann Neurol 1977;2:460-5. 